\title{
Bayesian Speckle Noise Reduction Using The Discrete Wavelet Transform
}

\author{
Zhaohui Zeng and Ian Cumming \\ Dept. of Electrical and Computer Engineering \\ The University of British Columbia \\ Vancouver, BC, Canada V6T 1 Z4. \\ zhaohuiz@ece.ubc.ca ianc@ece.ubc.ca
}

\begin{abstract}
Speckle noise is an inherent property of SAR images. The discrete wavelet transform results in sub-band decompositions of SAR images which have Laplacian point statistics. We propose a Bayesian estimator to perform speckle reduction. We evaluate the speckle reduction results using two measurements: standarddeviation-to-mean ratio and target-to-clutter ratio. We show that good speckle reduction results can be achieved while maintaining the significant information of the SAR image. ${ }^{1}$
\end{abstract}

\section{Introduction}

Synthetic aperture radar (SAR) is very efficient instrument for obtaining remotely sensed images of the earth's surface. However, SAR imagery is degraded by a form of multiplicative noise known as speckle which is a result of the illumination by the coherent radar. Hence, speckle reduction is a necessary procedure before automatic image analysis can be performed.

The discrete wavelet transform (DWT) is an efficient technique for multi-resolution decomposition of images and has been used for speckle reduction and enhancement of SAR images [1]. Here, we propose a speckle reduction algorithm in the wavelet domain. It is different from existing wavelet coefficient thresholding methods in that we estimate the image scene underlying the speckle using Bayes' rule based on the statistical properties of the wavelet coefficients.

\section{Speckle Noise in SAR Images}

Speckle can be described as multiplicative noise, with a standard deviation equal to the pixel reflectivity value. For a digitized SAR image, we define $y(j, k)$ as the gray level (or the observed image magnitude) of the $(j, k) t h$ pixel of the image. Hence, the measured pixel level of a SAR image can be written as:

\footnotetext{
${ }^{1}$ Presented at the 1998 International Geoscience and Remote Sensing Symposium, IGARSS'98, Seattle, 6-10 July 1998.
}

$$
y=x e
$$

where $x$ is the desired pixel reflectivity and $e$ is the multiplicative noise. Arsenault and April [2] showed that for a logarithmically-transformed SAR image, the speckle is approximately Gaussian additive noise:

$$
\bar{y}=\bar{x}+\bar{e}
$$

where $\bar{y}=\ln (|y|), \bar{x}=\ln (|x|), \bar{e}=\ln (|e|)$.

Because the multi-level DWT is a linear transform, speckle noise is still additive in the wavelet domain. If $W$ is the multi-level DWT, then a multi-resolution representation is given by the equation:

$$
W \bar{y}=W \bar{x}+W \bar{e}(\text { or }, Y=X+E)
$$

where $Y=W \bar{y}, X=W \bar{x}, E=W \bar{e}$. The noise level (standard deviation), $\sigma$, in the multi-resolution representation is not known in advance and has to be estimated from the data. Here, an estimate of $\sigma$ is taken to be the standard derivation of the first level diagonal sub-band image because this sub-band is mainly noise [3].

\section{Bayesian Estimator}

Our methodology is to estimate the noise-cleaned wavelet coefficient $X$ given the measured $Y$. The mean of the posterior distribution provides an unbiased leastsquare estimate $\hat{X}$ of the variable $X$. The Bayes' rule allows us to write this in terms of the probability densities of the noise and signal:

$$
\begin{aligned}
\hat{X}(Y) & =\int P_{X \mid Y}(X \mid Y) X d X \\
& =\int \frac{P_{Y \mid X}(Y \mid X) P_{X}(X) X d X}{P_{Y \mid X}(Y \mid X) P_{X}(X) d X} \\
& =\int \frac{P_{E}(Y-X) P_{X}(X) X d X}{P_{E}(Y-X) P_{X}(X) d X}
\end{aligned}
$$

where $P_{E}$ indicates the probability density function of the noise, and $P_{X}$ the prior probability density function of the 


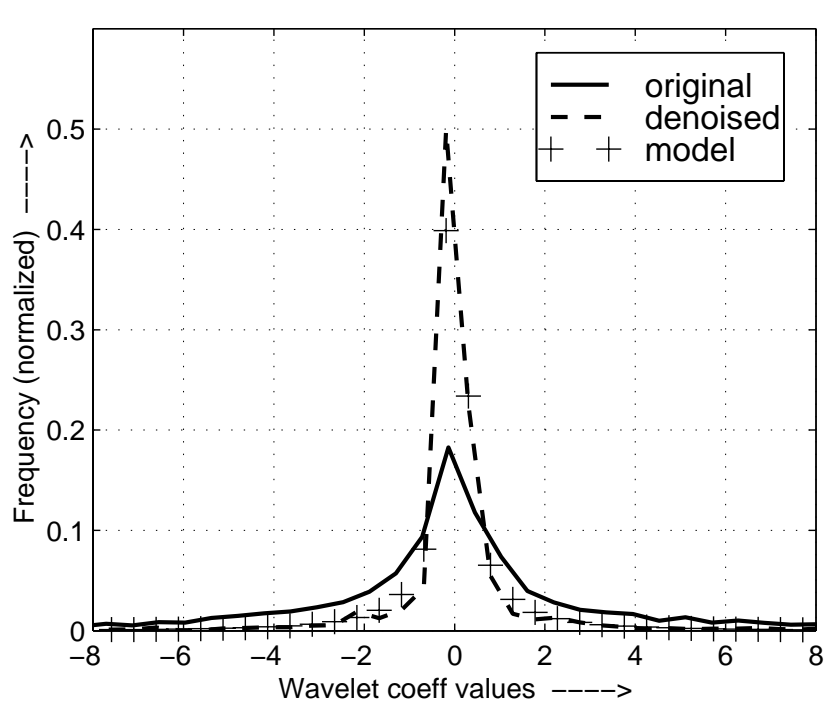

Figure 1: Histogram of second-level horizontal detail wavelet coefficients (see text).

signal. The denominator is the probability distribution functions (PDF) of the noisy observation, computed via convolution of the noise and signal pdf. In order to use this equation to estimate the original value $X$, we must know both of these probability density functions.

In [4], Mallat described wavelet coefficients (without speckle noise) as a two-parameter generalized Laplacian distribution:

$$
P_{X}(X)=\frac{e^{-|X / s|^{p}}}{N(s, p)}
$$

where $N(s, p)=2 s,(1 / p) / p$, and,$(X)=\int_{0}^{\infty} t^{X-1} e^{-t} d t$, the well known "gamma" function. The distribution is zero-mean and symmetric, and the parameters $\{s, p\}$ are directly related to the second and fourth moments of $\mathrm{X}$ :

$$
\sigma^{2}=\frac{s^{2},\left(\frac{3}{p}\right)}{,\left(\frac{1}{p}\right)}, \kappa=\frac{,\left(\frac{1}{p}\right),\left(\frac{5}{p}\right)}{{ }^{2}\left(\frac{3}{p}\right)}
$$

where $\kappa$ is the kurtosis (fourth moment divided by squared variance). Figure 1 gives the histogram of the wavelet coefficients of the second-level horizontal detail sub-band image. The plot shows the histogram of: a) the coefficients of the original image; b) the coefficients of the denoised image; and c) the coefficients given by the Laplacian distribution. This shows that the denoised image has much less high frequency energy than the original, and also that coefficients of the denoised image fit the Laplacian model well.

Also, we can estimate $\{s, p\}$ from the noisy observation $Y$. We find that the second and fourth moments of a generalized Laplacian signal corrupted by additive Gaussian white noise are:

$$
\sigma^{2}=\sigma_{n}^{2}+\frac{s^{2},\left(\frac{3}{p}\right)}{,\left(\frac{1}{p}\right)}
$$

$$
m_{4}=3 \sigma_{n}^{4}+\frac{6 \sigma_{n}^{2} s^{2},\left(\frac{3}{p}\right)}{,\left(\frac{1}{p}\right)}+\frac{s^{4},\left(\frac{5}{p}\right)}{,\left(\frac{1}{p}\right)}
$$

Assuming $\sigma_{n}$ is known, the measurements of these two moments of the noisy data are sufficient to estimate the model pdf parameters.

\section{Experimental Results}

In our experiments, Daubechies 4 (DB4) wavelet transforms (quadrature mirror filter pairs) are employed. We construct a 3-level wavelet decomposition. For each wavelet sub-band, we estimate the model pdf parameters $\mathrm{S}$ and $\mathrm{P}$, and numerically compute an estimate via equation (6). After applying this estimator, we reconstruct $\hat{x}$ from the inverse discrete wavelet transform (IDWT) of $\hat{X}$ without further coefficient quantization.

Figure 2 is X-band high-resolution airborne 1-look SAR image showing part of Bedfordshire in south-east England. It displays many of the features that typically appear in airborne imagery. The scene selected as the test image for our algorithm is shown in the lower left-hand corner.

Two statistics are used to evaluate the performance of our algorithm:

- Standard-deviation-to-mean ratio $(\mathrm{s} / \mathrm{m})$ : is a measure of image speckle in homogeneous regions [5].

- Target-to-clutter ratio $(\mathbf{t} / \mathbf{c})$ : is the difference between the target and clutter means (in dB). It measures how the target stands out of the surrounding clutter.

The original and speckle-reduced sub-images are shown in Figure 3. We choose areas A, B to measure standarddeviation-to-mean ratio, and areas $\mathrm{C}, \mathrm{D}$ to measure targetto-clutter ratio. Table 1 show the values of these two performance measures for the original and processed image within the selected regions. The significant reductions of standard-deviation-to-mean ratio indicates that a significant amount of speckle has been removed while the sharp features are maintained, which suggests potential improvements for classification and recognition. Although the target-to-clutter ratio is decreased a little, the target can be still easily recognized.

\begin{tabular}{|c|c|c|c|c|}
\hline & \multicolumn{2}{|c|}{$s / m$} & \multicolumn{2}{c|}{$t / c(d B)$} \\
\hline & area A & area B & area C & area D \\
\hline Original Image & 0.269 & 0.287 & 35.5 & 35.8 \\
\hline De-noised Image & 0.144 & 0.169 & 35.2 & 35.3 \\
\hline
\end{tabular}

Table 1: De-noising performance

\section{Discussion}

The Bayesian speckle noise removal described above 


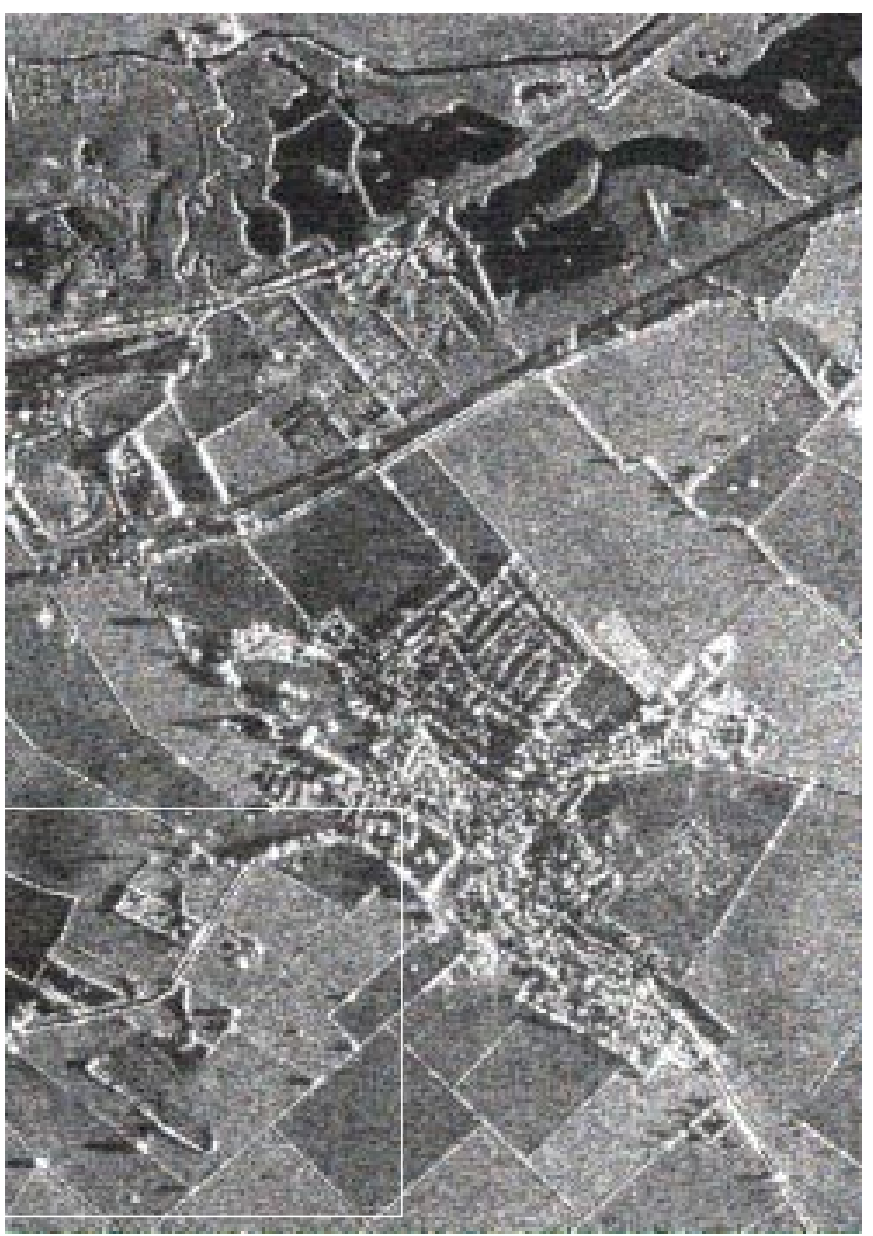

Figure 2: X-band SAR image Bedfordshire in southeast England (Provided by N. A. Software Ltd, http://www.nasofware.co.uk/).

provides a link between the statistical properties of the signal and the sub-band representation of the signal. The estimator is based on two key factors: sub-band decomposition and the statistical model of the wavelet coefficients. The quality of the noise removal can be improved by careful choice of the wavelet mother function and parameters for the pdf. In addition, joint statistical properties of the pixel and its spatial neighbors and sub-band neighbors can be taken into consideration to improve the result.

\section{References}

[1] D. Wei, H. Guo, J. Odegard, M. Lang, and C. Burrus, "Simultaneous speckle reduction and data compression using best wavelet packet bases with application to SAR based ATD/R", In SPIE conference on wavelet applications, volume 2491, Orlando, FL, April 1995.

[2] H. H. Arsenault and G. April, "Properties of speckle integrated with a finite aperture and logarithmically

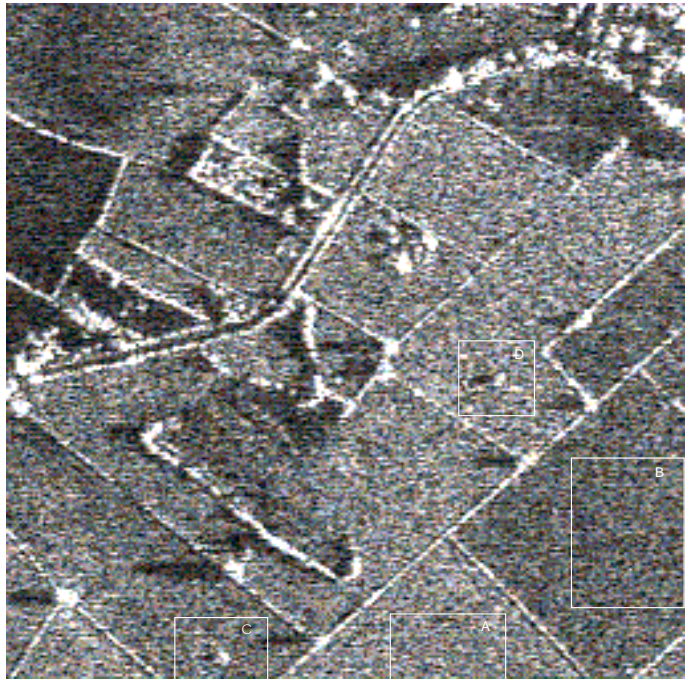

(a) Original image

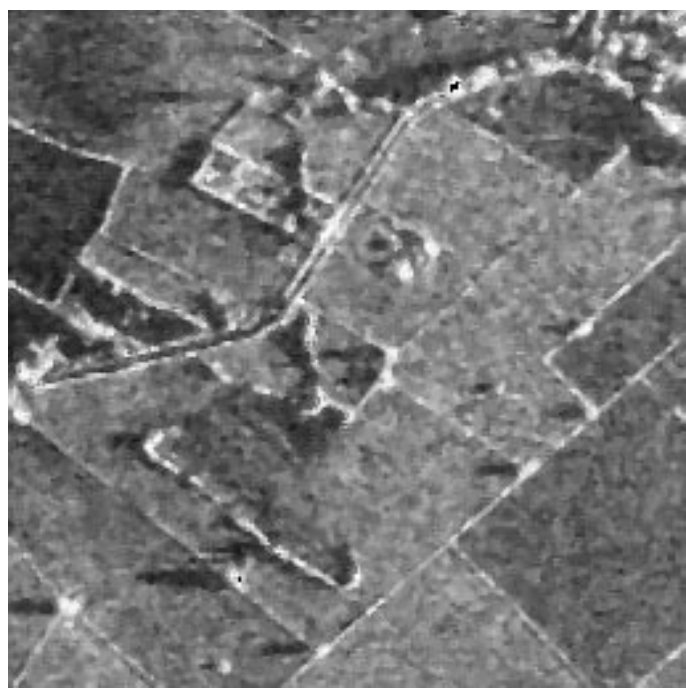

(b) De-noised image

Figure 3: Original and processed sub-images.

transformed", J. Opt. Soc. Am., vol. 66, pp. 11601163, Nov. 1976.

[3] D. Donoho, "De-noising by soft-thresholding", IEEE Trans. Inform. Theory, vol. IT-41, pp. 613-627, May 1995.

[4] S. G. Mallat, "A theory for multi-resolution signal decomposition", IEEE Trans. Pattern Anal. Machine Intel., vol. 11, pp. 674-693, 1989.

[5] J. W. Goodman, "Some fundamental properties of speckle", J. Opt. Soc. Am., 66:1145-1150, November 1976. 